\title{
Update As Evidence: Belief Expansion
}

\author{
Roman Kuznets` and Thomas Studer \\ Institut für Informatik und angewandte Mathematik \\ Universität Bern \\ \{kuznets, tstuder\}@iam.unibe.ch \\ http://www.iam.unibe.ch/ltg
}

\begin{abstract}
We introduce a justification logic with a novel constructor for evidence terms, according to which the new information itself serves as evidence for believing it. We provide a sound and complete axiomatization for belief expansion and minimal change and explain how the minimality can be graded according to the strength of reasoning. We also provide an evidential analog of the Ramsey axiom.
\end{abstract}

\section{Introduction}

Like modal logics, justification logics are epistemic logics that provide means to formalize properties of knowledge and belief. Modal logics use formulas $\square A$ to state that $A$ is known (or believed), where the modality $\square$ can be seen as an implicit knowledge operator since it does not provide any reason why $A$ is known. Justification logics operate with explicit evidence for an agent's knowledge using formulas of the form $t: A$ to state that $A$ is known for reason $t$. The evidence term $t$ may represent a formal mathematical proof of $A$ or an informal reason for believing $A$ such as a public announcement or direct observation of $A$.

Artemov developed the first justification logic, the Logic of Proofs, to give a classical provability semantics for intuitionistic logic [2-4]. In the area of formal epistemology, justification logics provide a novel approach to certain epistemic puzzles and problems of multiagent systems $[5-7,9,13]$.

The study of dynamic justification logics took off with Renne's PhD thesis [21] and his work on eliminating unreliable evidence [22]. He also investigated the expressive power of certain justification logics with announcements [23]. In a series of papers $[12,14,15]$ we examined two alternative justification counterparts of Gerbrandy-Groeneveld's public announcement logic [18]. Last but not least, Baltag et al. [10] introduced a justification logic for belief change, soft evidence, and defeasible knowledge.

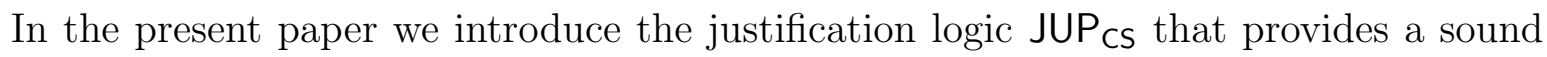
and complete axiomatization for belief expansion and minimal change. Our logic includes a new evidence term construct up $(A)$ that represents the update with $A$. Hence, after an update with $A$, the term up $(A)$ becomes a reason to believe $A$. Formally, this is modeled by the axiom $[A](\operatorname{up}(A): A)$.

In addition, the presence of explicit evidence makes it possible to axiomatize the principle of minimal change within the object language. For instance, in Lemma 20 we prove

\footnotetext{
* Supported by Swiss National Science Foundation grant PZ00P2-131706.
} 
that for each term $t$ that does not contain up $(A)$ as a subterm,

$$
\mathrm{JUP}_{\mathrm{CS}} \vdash \quad t: B \leftrightarrow[A](t: B)
$$

The direction from left to right is the persistence principle saying that we deal only with belief expansion. The direction from right to left states that if after an update with $A$ an agent believes $B$ for a reason that is independent from the update, then before the update the agent already believed $B$ for the same reason. Note that a principle of this kind cannot be formulated in a purely modal logic language. When $[A] \square B$ holds, it is not clear whether $\square B$ should be the case since it is not known whether the belief in $B$ depends on the update or is due to another, unrelated reason.

\section{The Logic JUPCS}

We start with countably many constants $c_{i}$, countably many variables $x_{i}$, and countably many atomic propositions $P_{i}$. The (evidence) terms and formulas of the language of JUP are defined as follows:

- Evidence terms.

- Every constant $c_{i}$ and every variable $x_{i}$ is an atomic term. If $A$ is a formula, then $\operatorname{up}(A)$ is an atomic term. Every atomic term is a term.

- If $t$ and $s$ are terms and $A$ is a formula, then $\left(t \cdot{ }_{A} s\right)$ is a term.

- Formulas.

- Every atomic proposition $P_{i}$ is a formula.

- If $A$ and $B$ are formulas, $\Gamma$ is a finite set of formulas, and $t$ is a term, then $\neg A$, $(A \rightarrow B), t: A$, and $[\Gamma] A$ are formulas.

We write $\left[A_{1}, \ldots, A_{n}\right] B$ instead of $\left[\left\{A_{1}, \ldots, A_{n}\right\}\right] B$, usually assuming all $A_{i}$ 's to be pairwise distinct.

ATm, Prop, Tm, and Fml denote the set of atomic terms, the set of atomic propositions, the set of evidence terms, and the set of formulas respectively. A formula $t$ : $A$ means that $A$ is believed for reason $t$ and $[\Gamma] A$ stands for $A$ holds after an update with all formulas in $\Gamma$. As usual, we define $(A \wedge B):=\neg(A \rightarrow \neg B)$ and $(A \leftrightarrow B):=((A \rightarrow B) \wedge(B \rightarrow A))$. We employ the standard conventions on the omission of brackets and postulate that both the colon operator in $t: A$ and the update operator in $[\Gamma] A$ bind stronger than any Boolean connective.

The set of axioms of JUP can be found in Fig. 1. Note that $\Gamma \cup \Delta$ in the axiom (It) is a finite set of formulas whenever $\Gamma$ and $\Delta$ are. The axioms (Taut) and (App) become the usual axioms of the justification logic $\mathrm{J}$ (see [6]) if (App) is formulated as an implication instead of the equivalence above. The present version with the equivalence yields a justification logics with minimal evidence. Later, when we define the semantics, this will correspond to the fact that the evidence relation is the least fixed point. This also explains why we need to annotate the application operator - by a formula: otherwise we would not be able to formulate the direction from right to left. Renne [22] was the first to use this 
1. all propositional tautologies

2. $t:(A \rightarrow B) \wedge s: A \leftrightarrow t \cdot A s: B$

3. $[\Gamma] P \leftrightarrow P$

4. $[\Gamma] \neg B \leftrightarrow \neg[\Gamma] B$

5. $[\Gamma](B \rightarrow C) \leftrightarrow([\Gamma] B \rightarrow[\Gamma] C)$

(Red.2)

6. $t: B \rightarrow[\Gamma] t: B$

(Red.3)

7. $\neg \operatorname{up}(A): B$

8. $[\Gamma] \operatorname{up}(A): A \quad$ if $A \in \Gamma$

9. $[\Gamma] t: A \rightarrow t: A$ if $t \in \mathrm{ATm}$ and either $t \neq \mathrm{up}(A)$ or $A \notin \Gamma$

Fig. 1. Axioms of JUP

kind of annotation. Axioms (Red.1)-(Red.3) are called reduction axioms. They make it possible to reduce the situation after an update occurred to the situation before the update. For instance, (Red.1) states that atomic facts are not affected by updates. Axiom (Pers) postulates that beliefs are persistent, i.e., that no contraction takes place because of an update and, consequently, the belief set can only be expanded. The update axiom (Up) claims that updates are introspectively successful: after an update with a formula $A$, the agent believes $A$ and the term up $(A)$ represents a reason for that belief, which is the update itself. The axiom (Init) postulates the special status of terms up $(A)$, which initially, i.e., before any updates, cannot serve as a basis for belief in anything. Axioms (MC.1) and (MC.2) formalize the principle of minimal change: an update should only lead to the smallest necessary change in the belief set. That means only those beliefs should be added that "logically" follow from the update and from what is already believed before. An interesting feature of our system is that the strength of the logic used for the deductive closure can be regulated. Finally, axiom (It) explains how to deal with iterated updates.

A constant specification CS (for JUP) is any subset

$$
\begin{aligned}
\mathrm{CS} \subseteq\left\{\left(c, \quad c_{1}: c_{2}: \ldots: c_{n}: A\right) \mid\right. \\
\left.n \geq 0, c, c_{1}, c_{2}, \ldots, c_{n} \text { are constants, and } A \text { is an axiom of JUP }\right\} .
\end{aligned}
$$

For a constant specification CS the deductive system JUP ${ }_{C S}$ is the Hilbert system given by the axioms of JUP and by the rules modus ponens and axiom necessitation:

$$
\frac{A \quad A \rightarrow B}{B}(\mathrm{MP}), \quad \frac{(c, B) \in \mathrm{CS}}{c: B}(\mathrm{AN}) .
$$

We write $J_{\text {US }} \vdash A$ if the formula $A$ is derivable in JUPCS.

We are now going to introduce a semantics for JUPCS that uses basic modular models. Artemov [8] introduced them for the basic justification logic $\mathrm{J}$ in order to provide an ontologically transparent semantics for justifications. Kuznets and Studer [19] later extended this construction to all justification counterparts of the logics from the modal cube between $\mathrm{K}$ and $\mathrm{S} 5$. The very first semantics of this kind, however, was presented by Mkrtychev [20]. 
Definition 1 (Evidence closure). Let $\mathcal{B} \subseteq \mathrm{ATm} \times \mathrm{Fml}$. For a set $X \subseteq \mathrm{Tm} \times \mathrm{Fml}$ we define $\mathrm{cl}_{\mathcal{B}}(X)$ by:

1. if $(t, A) \in \mathcal{B}$, then $(t, A) \in \mathrm{cl}_{\mathcal{B}}(X)$;

2. if $(s, A) \in X$ and $(t, A \rightarrow B) \in X$, then $\left(t \cdot{ }_{A} s, B\right) \in \mathrm{cl}_{\mathcal{B}}(X)$.

Note that $\mathrm{cl}_{\mathcal{B}}$ is a monotone operator on $\mathrm{Tm} \times \mathrm{Fml}$, that is

$$
X \subseteq Y \text { implies } \quad \operatorname{cl}_{\mathcal{B}}(X) \subseteq \mathrm{cl}_{\mathcal{B}}(Y)
$$

for all $X, Y \subseteq \mathrm{Tm} \times \mathrm{Fml}$. Hence, $\mathrm{cl}_{\mathcal{B}}$ has a least fixed point, which is shown as usual, see, e.g., [11].

Lemma 2 (Least fixed point). There is a unique $R \subseteq \mathrm{Tm} \times \mathrm{Fml}$ such that

1. $\mathrm{cl}_{\mathcal{B}}(R)=R$,

2. for any $S \subseteq \mathrm{Tm} \times \mathrm{Fml}$, if $\mathrm{cl}_{\mathcal{B}}(S) \subseteq S$, then $R \subseteq S$.

Proof. Let $C:=\left\{S \subseteq \mathrm{Tm} \times \mathrm{Fml} \mid \mathrm{cl}_{\mathcal{B}}(S) \subseteq S\right\}$. Since $\mathrm{Tm} \times \mathrm{Fml} \in C$, we know that $C$ is non-empty. Let $R:=\bigcap C$. The second claim now holds by definition. And the uniqueness of $R$ is an easy corollary of the second claim.

It remains to establish $\operatorname{cl}_{\mathcal{B}}(R)=R$. Let $S \in C$. Since $R \subseteq S$ and $\mathrm{cl}_{\mathcal{B}}$ is monotone, we find $\mathrm{cl}_{\mathcal{B}}(R) \subseteq \mathrm{cl}_{\mathcal{B}}(S)$. We also have $\mathrm{cl}_{\mathcal{B}}(S) \subseteq S$, so $\mathrm{cl}_{\mathcal{B}}(R) \subseteq S$. Since $S$ is an arbitrary element of $C$ and $R=\bigcap C$, this implies $\operatorname{cl}_{\mathcal{B}}(R) \subseteq R$.

To show $R \subseteq \mathrm{cl}_{\mathcal{B}}(R)$, we first observe that since $\mathrm{cl}_{\mathcal{B}}(R) \subseteq R$, we have $\operatorname{cl}_{\mathcal{B}}\left(\mathrm{cl}_{\mathcal{B}}(R)\right) \subseteq$ $\mathrm{cl}_{\mathcal{B}}(R)$ by monotonicity. Thus $\mathrm{cl}_{\mathcal{B}}(R) \in C$, which gives us $R \subseteq \mathrm{cl}_{\mathcal{B}}(R)$ because $R=\bigcap C$.

Definition 3 (Evidence relation). Let $\mathcal{B} \subseteq \mathrm{ATm} \times \mathrm{Fml}$. We define the minimal evidence relation $\mathcal{E}(\mathcal{B})$ as the least fixed point of $\mathrm{cl}_{\mathcal{B}}$.

It follows directly from the definition of $\mathrm{cl}_{\mathcal{B}}$ that

Lemma 4 (Properties of fixed points of $\mathrm{cl}_{\mathcal{B}}$ ). For any $\mathcal{B} \subseteq \mathrm{ATm} \times \mathrm{Fml}$ and any fixed point $F$ of $\mathrm{cl}_{\mathcal{B}}$, e.g., for $F=\mathcal{E}(\mathcal{B})$ :

1. $(t, A) \in F \quad$ iff $\quad(t, A) \in \mathcal{B} \quad$ for any $t \in \mathrm{ATm}$.

2. $\left(t \cdot{ }_{A} s, B\right) \in F \quad$ iff $(t, A \rightarrow B) \in F$ and $(s, A) \in F$.

Further, we get the following lemma.

Lemma 5 (Monotonicity of $\mathcal{E}$ ). Let $\mathcal{B}, \mathcal{C} \subseteq \mathrm{ATm} \times \mathrm{Fml}$. We have that $\mathcal{E}(\mathcal{B}) \subseteq \mathcal{E}(\mathcal{B} \cup \mathcal{C})$.

Proof. By induction on $t$ we show $(t, A) \in \mathcal{E}(\mathcal{B})$ implies $(t, A) \in \mathcal{E}(\mathcal{B} \cup \mathcal{C})$ for all formulas $A$. Assume $(t, A) \in \mathcal{E}(\mathcal{B})$. We have one of the following cases.

1. $t \in$ ATm. Then $(t, A) \in \mathcal{B}$ by Lemma 4.1. Since $(t, A) \in \mathcal{B} \cup \mathcal{C}$, it follows from the same lemma that $(t, A) \in \mathcal{E}(\mathcal{B} \cup \mathcal{C})$. 
2. $t=r \cdot_{B} s$. Then $\{(s, B),(r, B \rightarrow A)\} \subseteq \mathcal{E}(\mathcal{B})$ by Lemma 4.2. By IH we find $\{(s, B),(r, B \rightarrow$ $A)\} \subseteq \mathcal{E}(\mathcal{B} \cup \mathcal{C})$. We get $(t, A)=\left(r \cdot_{B} s, A\right) \in \mathcal{E}(\mathcal{B} \cup \mathcal{C})$ by Lemma 4.2.

Definition 6 (Model, initial model, updated model). $A$ model is a pair $\mathcal{M}=(\mathrm{v}, \mathcal{B})$ where $\mathrm{v} \subseteq$ Prop and $\mathcal{B} \subseteq \mathrm{ATm} \times \mathrm{Fml}$. For a constant specification $\mathrm{CS}$, the model $\mathcal{M}$ is called a CS-model if $\mathrm{CS} \subseteq \mathcal{B}$. The model $\mathcal{M}$ is called initial if $(\operatorname{up}(A), B) \notin \mathcal{B}$ for any formulas $A$ and $B$.

For a finite set $\Gamma$ of formulas, the updated model $\mathcal{M}^{\Gamma}:=\left(\mathrm{v}, \mathcal{B}^{\Gamma}\right)$ is defined by $\mathcal{B}^{\Gamma}:=$ $\mathcal{B} \cup \mathcal{U}_{\Gamma}$ with $\mathcal{U}_{\Gamma}:=\{(\operatorname{up}(A), A) \mid A \in \Gamma\}$. For a singleton set $\Gamma=\{A\}$ we write $\mathcal{M}^{A}$ and $\mathcal{B}^{A}$ instead of $\mathcal{M}^{\{A\}}$ and $\mathcal{B}^{\{A\}}$ respectively.

Remark 7. Note that our definition of a model update is independent of which formulas are true, unlike Plaza-style, Gerbrandy-Groeneveld-style, or action model-style updates, where the definitions of model update and truth in the model have to be given by simultaneous induction. This ontological separation of reasons for belief from truth is inherent in Artemov's semantics of modular models [8], which we adopt and adapt in this paper.

\section{Lemma 8 (Properties of updated models).}

1. $\mathcal{M}^{\varnothing}=\mathcal{M}$,

2. $\left(\mathcal{M}^{\Gamma}\right)^{\Delta}=\mathcal{M}^{\Gamma \cup \Delta}$,

3. For any constant specification $\mathrm{CS}$, any $\mathrm{CS}$-model $\mathcal{M}$, and any finite set $\Gamma$ of formulas, the model $\mathcal{M}^{\Gamma}$ is a CS-model.

Proof. Immediately follows from $\mathcal{U}_{\varnothing}=\varnothing, \mathcal{U}_{\Gamma} \cup \mathcal{U}_{\Delta}=\mathcal{U}_{\Gamma \cup \Delta}$, and $\mathcal{B} \subseteq \mathcal{B}^{\Gamma}$ respectively.

Definition 9 (Truth). Let $\mathcal{M}=(\mathrm{v}, \mathcal{B})$ be a model and $D$ be a formula. We define the relation $\mathcal{M} \Vdash D$ by

1. $\mathcal{M} \Vdash P \quad$ iff $P \in \mathrm{v}$

2. $\mathcal{M} \Vdash \neg A \quad$ iff $\mathcal{M} \nVdash A$

3. $\mathcal{M} \Vdash A \rightarrow B \quad$ iff $\mathcal{M} \nVdash A$ or $\mathcal{M} \Vdash B$

4. $\mathcal{M} \Vdash t: A \quad$ iff $(t, A) \in \mathcal{E}(\mathcal{B})$

5. $\mathcal{M} \Vdash[\Gamma] A \quad$ iff $\quad \mathcal{M}^{\Gamma} \Vdash A$.

A formula $D$ is valid with respect to a constant specification CS if $\mathcal{M} \Vdash D$ for all initial CS-models $\mathcal{M}$.

\section{Soundness}

For this section and the next one, we assume CS to be a fixed but arbitrary constant specification. In later sections, the use of soundness and completeness with respect to models with no CS specified should be understood as soundness and completeness with respect to initial $\varnothing$-models because any model is an $\varnothing$-model.

Theorem 10 (Soundness). For all formulas D,

$$
\text { JUP }_{\mathrm{CS}} \vdash D \text { implies } D \text { is valid with respect to CS . }
$$


Proof. As usual the proof is by induction on the length of the derivation of $D$. Let $\mathcal{M}=$ $(\mathrm{v}, \mathcal{B})$ be an initial CS-model.

1. (Taut). All instances of propositional tautologies hold under $\mathcal{M}$.

2. (App). $\mathcal{M} \Vdash t:(A \rightarrow B) \wedge s: A$ iff $\{(t, A \rightarrow B),(s, A)\} \subseteq \mathcal{E}(\mathcal{B})$. By Lemma 4.2 , this is equivalent to $\left(t \cdot \cdot_{A} s, B\right) \in \mathcal{E}(\mathcal{B})$, in other words to $\mathcal{M} \Vdash t \cdot{ }_{A} s: B$.

3. (Red.1). $\mathcal{M} \Vdash[\Gamma] P$ iff $\mathcal{M}^{\Gamma} \Vdash P$ iff $P \in \mathrm{v}$ iff $\mathcal{M} \Vdash P$.

4. (Red.2). $\mathcal{M} \Vdash[\Gamma] \neg B$ iff $\mathcal{M}^{\Gamma} \Vdash \neg B$ iff $\mathcal{M}^{\Gamma} \nVdash B$ iff $\mathcal{M} \nVdash[\Gamma] B$ iff $\mathcal{M} \Vdash \neg[\Gamma] B$.

5. (Red.3). Similar to the previous case.

6. (Pers). Follows immediately from Lemma 5.

7. (Init). $(\operatorname{up}(A), B) \notin \mathcal{B}$ since $\mathcal{M}$ is initial. $(\operatorname{up}(A), B) \notin \mathcal{E}(\mathcal{B})$ by Lemma 4.1. Thus, $\mathcal{M} \Vdash \neg \operatorname{up}(A): B$.

8. (Up). If $A \in \Gamma$, then $(\operatorname{up}(A), A) \in \mathcal{U}_{\Gamma} \subseteq \mathcal{B}^{\Gamma}$, and $(\operatorname{up}(A), A) \in \mathcal{E}\left(\mathcal{B}^{\Gamma}\right)$ by Lemma 4.1. It follows that $\mathcal{M}^{\Gamma} \Vdash \operatorname{up}(A): A$ and $\mathcal{M} \Vdash[\Gamma] \mathrm{up}(A): A$.

9. (MC.1). Assume $\mathcal{M} \Vdash[\Gamma] t: A$ for $t \in \mathrm{ATm}$ such that either $t \neq \operatorname{up}(A)$ or $A \notin \Gamma$. Then $\mathcal{M}^{\Gamma} \Vdash t: A$ and $(t, A) \in \mathcal{E}\left(\mathcal{B}^{\Gamma}\right)$. Since $t \in A T m$, we get $(t, A) \in \mathcal{B}^{\Gamma}=\mathcal{B} \cup \mathcal{U}_{\Gamma}$ by Lemma 4.1. Clearly, $(t, A) \notin \mathcal{U}_{\Gamma}$. Hence, $(t, A) \in \mathcal{B}$, and $(t, A) \in \mathcal{E}(\mathcal{B})$ by Lemma 4.1. Therefore, we conclude that $\mathcal{M} \Vdash t: A$.

10. (MC.2). Similar to Case 2 but for $\mathcal{M}^{\Gamma}$.

11. (It). $\mathcal{M} \Vdash[\Gamma][\Delta] A$ iff $\mathcal{M}^{\Gamma} \Vdash[\Delta] A$ iff $\left(\mathcal{M}^{\Gamma}\right)^{\Delta} \Vdash A$. By Lemma $8,\left(\mathcal{M}^{\Gamma}\right)^{\Delta}=\mathcal{M}^{\Gamma \cup \Delta}$. Thus, the equivalence continues as $\mathcal{M}^{\Gamma \cup \Delta} \Vdash A$ iff $\mathcal{M} \Vdash[\Gamma \cup \Delta] A$.

12. (MP). It is trivial to see that modus ponens preserves truth in a model.

13. (AN). For any $(c, B) \in \mathrm{CS}$, we have $(c, B) \in \mathcal{B}$ by definition of a CS-model. Further, $(c, B) \in \mathcal{E}(\mathcal{B})$ by Lemma 4.1 , and $\mathcal{M} \Vdash c: B$.

\section{Completeness}

Definition 11 (Consistency). A set $\Phi$ of formulas, finite or infinite, is called consistent if $\mathrm{JUP}_{\mathrm{CS}} \nvdash \neg\left(A_{1} \wedge \cdots \wedge A_{n}\right)$ for any finite subset $\left\{A_{1}, \ldots, A_{n}\right\} \subseteq \Phi$.

$A$ set $\Phi$ is called maximal consistent if it is consistent whereas no proper superset of $\Phi$ is.

Definition 12 (Induced model). Let $\Phi$ be a maximal consistent set of formulas. The model $\mathcal{M}_{\Phi}=\left(\mathrm{v}_{\Phi}, \mathcal{B}_{\Phi}\right)$ that is induced by $\Phi$ is given by

1. $P \in \mathrm{v}_{\Phi} \quad$ iff $P \in \Phi \cap$ Prop.

2. $(t, A) \in \mathcal{B}_{\Phi} \quad$ iff $t \in \mathrm{ATm}$ and $t: A \in \Phi$.

$\mathcal{M}_{\Phi}$ is an initial CS-model. Indeed, by the maximal consistency of $\Phi,(c, B) \in \mathcal{B}_{\Phi}$ since $c: B \in \Phi$ since $J_{\text {UP }} \vdash c: B$ for every $(c, B) \in \mathrm{CS}$ and $(\operatorname{up}(A), B) \notin \mathcal{B}_{\Phi}$ since up $(A): B \notin \Phi$ since $\neg$ up $(A): B \in \Phi$ since JUP cs $\vdash \neg$ up $(A): B$ for arbitrary $A$ and $B$.

Lemma 13 (Canonical evidence). Let $\Phi$ be a maximal consistent set. Then

$$
t: A \in \Phi \quad \Longleftrightarrow \quad(t, A) \in \mathcal{E}\left(\mathcal{B}_{\Phi}\right)
$$


Proof. By induction on $t$.

1. $t \in$ ATm. We have $t: A \in \Phi$ iff (by definition) $(t, A) \in \mathcal{B}_{\Phi}$ iff (by Lemma 4.1) $(t, A) \in$ $\mathcal{E}\left(\mathcal{B}_{\Phi}\right)$.

2. $t=r \cdot{ }_{B} s$. We have $r \cdot{ }_{B} s: A \in \Phi$ iff (by (App) and maximal consistency of $\left.\Phi\right)\{s: B, r:(B \rightarrow$ $A)\} \subseteq \Phi$ iff (by IH) $\{(s, B),(r, B \rightarrow A)\} \subseteq \mathcal{E}\left(\mathcal{B}_{\Phi}\right)$ iff (by Lemma 4.2) $\left(r \cdot_{B} s, A\right) \in \mathcal{E}\left(\mathcal{B}_{\Phi}\right)$.

Definition 14 (Rank). We inductively define the rank of a term by

1. $\operatorname{rk}(t):=1 \quad$ if $t \in \mathrm{ATm}$;

2. $\operatorname{rk}\left(s \cdot{ }_{A} t\right):=\max (\mathrm{rk}(s), \mathrm{rk}(t))+1$;

and the rank of a formula by

1. $\operatorname{rk}(P):=1 \quad$ if $P \in \operatorname{Prop}$;

2. $\operatorname{rk}(\neg A):=\operatorname{rk}(A)+1$;

3. $\operatorname{rk}(A \rightarrow B):=\max (\operatorname{rk}(A), \operatorname{rk}(B))+1$;

4. $\operatorname{rk}(t: A):=\operatorname{rk}(t)$;

5. $\operatorname{rk}([\Gamma] B):=2 \cdot \operatorname{rk}(B)$.

We immediately get the following properties of rk.

\section{Lemma 15 (Reduction).}

1. $\operatorname{rk}([\Gamma] A)>\operatorname{rk}(A)$.

2. $\operatorname{rk}([\Gamma] \neg B)>\operatorname{rk}(\neg[\Gamma] B)$.

3. $\operatorname{rk}([\Gamma](A \rightarrow B))>\operatorname{rk}([\Gamma] A \rightarrow[\Gamma] B)$.

4. $\operatorname{rk}\left([\Gamma] r \cdot{ }_{C} s: B\right)>\operatorname{rk}([\Gamma] r:(C \rightarrow B))$ and $\operatorname{rk}([\Gamma] r \cdot C s: B)>\operatorname{rk}([\Gamma] s: C)$.

5. $\operatorname{rk}([\Gamma][\Delta] A)>\operatorname{rk}([\Gamma \cup \Delta] A)$.

Lemma 16 (Truth lemma). Let $\Phi$ be a maximal consistent set of formulas. Then

$$
A \in \Phi \quad \Longleftrightarrow \mathcal{M}_{\Phi} \Vdash A
$$

Proof. By induction on rk $(A)$.

1. $A \in$ Prop. We have $A \in \Phi$ iff (by definition) $A \in \mathrm{v}_{\Phi}$ iff (by definition) $\mathcal{M}_{\Phi} \Vdash A$.

2. $A=\neg B$. We have $\neg B \in \Phi$ iff (by maximal consistency of $\Phi$ ) $B \notin \Phi$ iff (by IH) $\mathcal{M}_{\Phi} \nVdash B$ iff $\mathcal{M}_{\Phi} \Vdash \neg B$.

3. $A=B \rightarrow C$. We have $B \rightarrow C \in \Phi$ iff (by maximal consistency of $\Phi) B \notin \Phi$ or $C \in \Phi$ iff (by IH) $\mathcal{M}_{\Phi} \nVdash B$ or $\mathcal{M}_{\Phi} \Vdash C$ iff $\mathcal{M}_{\Phi} \Vdash B \rightarrow C$.

4. $A=t: B$. We have $t: B \in \Phi$ iff (by Lemma 13) $(t, B) \in \mathcal{E}\left(\mathcal{B}_{\Phi}\right)$ iff (by definition) $\mathcal{M}_{\Phi} \Vdash t: B$.

5. $A=[\Gamma] P$. We have $[\Gamma] P \in \Phi$ iff (by (Red.1) and maximal consistency of $\Phi) P \in \Phi$ iff (by IH) $\mathcal{M}_{\Phi} \Vdash P$ iff (by (Red.1) and soundness) $\mathcal{M}_{\Phi} \Vdash[\Gamma] P$. 
6. $A=[\Gamma] \neg B$. Then $[\Gamma] \neg B \in \Phi$ iff (by (Red.2) and maximal consistency of $\Phi) \neg[\Gamma] B \in \Phi$ iff (by IH) $\mathcal{M}_{\Phi} \Vdash \neg[\Gamma] B$ iff (by (Red.2) and soundness) $\mathcal{M}_{\Phi} \Vdash[\Gamma] \neg B$.

7. $A=[\Gamma](B \rightarrow C)$. We have $[\Gamma](B \rightarrow C) \in \Phi$ iff (by (Red.3) and maximal consistency of $\Phi)[\Gamma] B \rightarrow[\Gamma] C \in \Phi$ iff (by IH) $\mathcal{M}_{\Phi} \Vdash[\Gamma] B \rightarrow[\Gamma] C$ iff (by (Red.3) and soundness) $\mathcal{M}_{\Phi} \Vdash[\Gamma](B \rightarrow C)$.

8. $A=[\Gamma] t: B$. We distinguish the following cases for $t$.

(a) $t \in$ ATm. There are two possibilities:

$-t=\operatorname{up}(B)$ and $B \in \Gamma$. In this case, $A=[\Gamma]$ up $(B): B$ is an axiom. Therefore, we have $A \in \Phi$ by maximal consistency of $\Phi$ and $\mathcal{M}_{\Phi} \Vdash A$ by soundness;

- either $t \neq \operatorname{up}(B)$ or $B \notin \Gamma$. We have that $[\Gamma] t: B \in \Phi$ iff (by (Pers), (MC.1), and maximal consistency of $\Phi) t: B \in \Phi$ iff (by IH) $\mathcal{M}_{\Phi} \Vdash t: B$ iff (by (Pers), (MC.1), and soundness) $\mathcal{M}_{\Phi} \Vdash[\Gamma] t: B$.

(b) $t=r \cdot{ }_{C} s$. We have $[\Gamma] r \cdot{ }_{C} s: B \in \Phi$ iff (by (MC.2) and maximal consistency of $\Phi$ ) $\{[\Gamma] r:(C \rightarrow B),[\Gamma] s: C\} \subseteq \Phi$ iff $($ by $\mathrm{IH}) \mathcal{M}_{\Phi} \Vdash[\Gamma] r:(C \rightarrow B) \wedge[\Gamma] s: C$ iff (by (MC.2) and soundness) $\mathcal{M}_{\Phi} \Vdash[\Gamma] r \cdot{ }_{C} s: B$.

9. $A=[\Gamma][\Delta] B$. Then $[\Gamma][\Delta] B \in \Phi$ iff (by (It) and maximal consistency of $\Phi)[\Gamma \cup \Delta] B \in \Phi$ iff (by IH) $\mathcal{M}_{\Phi} \Vdash[\Gamma \cup \Delta] B$ iff (by (lt) and soundness) $\mathcal{M}_{\Phi} \Vdash[\Gamma][\Delta] B$.

Theorem 17 (Completeness). For all formulas D,

$$
D \text { is valid with respect to CS implies } \operatorname{JUP}_{\mathrm{CS}} \vdash D \text {. }
$$

Proof. Assume JUPCS $\nvdash D$. Then $\{\neg D\}$ is consistent and, hence, contained in a maximal consistent set $\Phi$. By the previous lemma we find $\mathcal{M}_{\Phi} \Vdash \neg D$. Thus, we conclude $\mathcal{M}_{\Phi} \nVdash D$, which means that $D$ is not valid with respect to CS since $\mathcal{M}_{\Phi}$ is an initial CS-model.

We now show that the update with the empty set $\Gamma=\varnothing$, i.e., the update with no additional information, has no effect.

Lemma 18. JUP ${ }_{\mathrm{CS}} \vdash[\varnothing] A \leftrightarrow A$.

Proof. Follows from $\mathcal{M}^{\varnothing}=\mathcal{M}$ (Lemma 8) and completeness.

We show the following principle of minimal change: an update with $A$ has no effect on beliefs that are justified without reference to that update.

Definition 19 (Subterms). Subterms of a term $t$ are defined by induction as follows. $S u b(t):=\{t\}$ if $t \in \mathrm{ATm}$.

$$
S u b\left(t \cdot{ }_{A} s\right):=S u b(t) \cup S u b(s) \cup\left\{t \cdot{ }_{A} s\right\} .
$$

Lemma 20 (Minimal change). Let $\Gamma$ be a finite set of formulas and $t$ be a term that does not contain up $(A)$ as a subterm for any $A \in \Gamma$. Then JUPCS $\vdash[\Gamma] t: B \leftrightarrow t: B$.

Proof. The direction from right to left follows from (Pers). To show the other direction, let $\mathcal{M}=(\mathrm{v}, \mathcal{B})$ be an initial CS-model with $\mathcal{M} \Vdash[\Gamma] t: B$. We prove $\mathcal{M} \Vdash t: B$ by induction on the structure of $t$. 
1. $t \in \mathrm{ATm}$. Then, $(t, B) \in \mathcal{B}^{\Gamma}=\mathcal{B} \cup\{(\operatorname{up}(A), A) \mid A \in \Gamma\}$ by Lemma 4.1. Since $t \neq \operatorname{up}(A)$ for any $A \in \Gamma$, we find $(t, B) \in \mathcal{B}$. Thus, $(t, B) \in \mathcal{E}(\mathcal{B})$ by Lemma 4.1, and $\mathcal{M} \Vdash t: B$ follows.

2. $t=r \cdot{ }_{C} s$. Then $\mathcal{M} \Vdash[\Gamma] s: C \wedge[\Gamma] r:(C \rightarrow B)$ by (MC.2) and soundness. By IH we find $\mathcal{M} \Vdash s: C \wedge r:(C \rightarrow B)$. Thus, we conclude by (App) and soundness that $\mathcal{M} \Vdash t: B$.

The claim follows by completeness.

\section{$5 \quad$ AGM Postulates}

In the now classic paper [1], Alchourrón, Gärdenfors, and Makinson introduced their famous postulates for belief contraction and revision where the underlying principle is that of minimal change. Later Gärdenfors [17] added postulates for belief expansion. We are going to show that the update operator of JUPCS satisfies these postulates for expansion, see Lemma 27.

Before we can state and prove Gärdenfors's postulates, we need to introduce the notion of a belief set and of the belief set induced by a model.

Definition 21 (Belief set). A belief set is a set $X \subseteq \mathrm{Fml}$ of formulas that satisfies

$$
\text { if } A \in X \text { and } A \rightarrow B \in X \text {, then } B \in X \text {. }
$$

Definition 22 (Induced beliefs). Let $\mathcal{M}$ be a model. We define the beliefs $\square_{\mathcal{M}}$ induced by $\mathcal{M}$ as

$$
\square_{\mathcal{M}}:=\{A \in \mathrm{Fml} \mid \mathcal{M} \Vdash t: A \text { for some } t \in \mathrm{Tm}\} \text {. }
$$

Lemma 23 (Induced beliefs). Let $\mathcal{M}$ be a model. Then $\square_{\mathcal{M}}$ is a belief set.

Proof. We have to show the condition of Definition 21. Assume that $A \in \square_{\mathcal{M}}$ and $A \rightarrow$ $B \in \square_{\mathcal{M}}$. Then there are terms $s$ and $t$ such that $\mathcal{M} \Vdash t: A$ and $\mathcal{M} \Vdash s:(A \rightarrow B)$. Therefore, $\mathcal{M} \Vdash s \cdot{ }_{A} t: B$ and hence $B \in \square_{\mathcal{M}}$.

Definition 24 (Expansion). Let $\mathcal{M}$ be a model and $A$ be a formula. We define

$$
\square_{\mathcal{M}} \oplus A:=\square_{\mathcal{M}^{A}}
$$

Definition 25. A constant specification CS is called

- propositionally appropriate (axiomatically appropriate) if for every $A$ that is a propositional tautology (an axiom of JUP) there exists a constant $c$ such that $(c, A) \in \mathrm{CS}$;

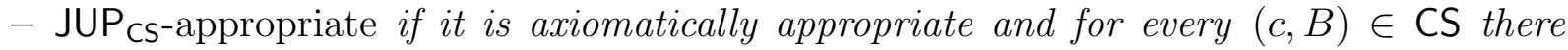
exists a constant $c^{\prime}$ such that $\left(c^{\prime}, c: B\right) \in \mathrm{CS}$.

Lemma 26. Let $\mathcal{M}$ be an initial CS-model. If CS is propositionally appropriate (axiomat-

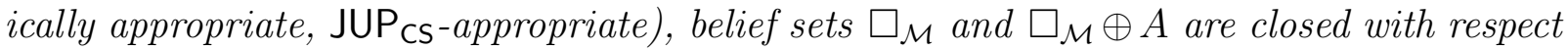
to classical propositional reasoning (reasoning in $\mathrm{JUP}_{\varnothing}$, reasoning in $\mathrm{JUP}_{\mathrm{CS}}$ ). 
Proof. What we need to prove is that whenever $C \in \square_{\mathcal{M}}\left(C \in \square_{\mathcal{M}} \oplus A\right)$ and $C \vdash_{T h} D$, it follows that $D \in \square_{\mathcal{M}}\left(D \in \square_{\mathcal{M}} \oplus A\right)$, where $T h$ stands for classical propositional logic in the language of JUP in the case of a propositionally appropriate CS, for JUP $\varnothing$ in the case

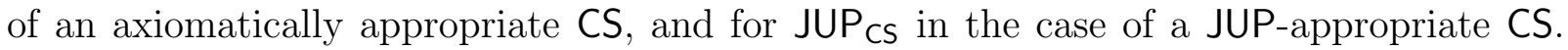
This can be easily demonstrated by induction on the derivation in the respective logic.

Lemma 27 (Postulates for expansion). Let $\mathcal{M}=(\mathrm{v}, \mathcal{B})$ be a model and $A$ be a formula. Then $X=\square_{\mathcal{M}} \oplus A$ satisfies the following properties:

1. $X$ is a belief set.

2. $A \in X$.

3. $\square_{\mathcal{M}} \subseteq X$.

Moreover, $\square_{\mathcal{M}} \oplus A$ is the smallest set satisfying Properties 1-3:

4. for any set $X \subseteq \mathrm{Fml}$ satisfying Properties 1-3 we have $\square_{\mathcal{M}} \oplus A \subseteq X$.

Proof. 1. Since Lemma 23 holds for arbitrary models, we immediately obtain that $\square_{\mathcal{M}} \oplus$ $A=\square_{\mathcal{M}^{A}}$ is a belief set.

2. By (Up) and soundness we have $\mathcal{M} \Vdash[A]$ up $(A): A$, in other words, $\mathcal{M}^{A} \Vdash \operatorname{up}(A): A$. Thus we get $A \in \square_{\mathcal{M}^{A}}$, i.e., $A \in \square_{\mathcal{M}} \oplus A$.

3. Assume $B \in \square_{\mathcal{M}}$. There exists a term $t$ such that $\mathcal{M} \Vdash t: B$. By (Pers) and soundness, we have $\mathcal{M} \Vdash[A] t: B$, in other words, $\mathcal{M}^{A} \Vdash t: B$. Thus, we get $B \in \square_{\mathcal{M}^{A}}$, i.e., $B \in \square_{\mathcal{M}} \oplus A$.

4. Let $X$ satisfy Properties $1-3$. We have to show $\square_{\mathcal{M}} \oplus A \subseteq X$. By the definition of $\square_{\mathcal{M}} \oplus$ $A$, this amounts to showing

$$
\mathcal{M}^{A} \Vdash t: B \text { implies } B \in X .
$$

Let $\mathcal{M}^{A}=\left(\mathrm{v}, \mathcal{B}^{A}\right)$. To establish (1) it is enough to show

$$
(t, B) \in \mathcal{E}\left(\mathcal{B}^{A}\right) \text { implies } B \in X \text {. }
$$

We establish (2) by induction on the structure of $t$. Assume $(t, B) \in \mathcal{E}\left(\mathcal{B}^{A}\right)$. We have one of the following cases:

(a) $t \in$ ATm. By Lemma 4.1, $(t, B) \in \mathcal{B}^{A}=\mathcal{B} \cup\{(\operatorname{up}(A), A)\}$. If $(t, B) \in \mathcal{B}$, then $(t, B) \in \mathcal{E}(\mathcal{B})$ by Lemma 4.1 , thus, $\mathcal{M} \Vdash t: B$, i.e., $B \in \square_{\mathcal{M}}$. By Property 3 for $X$, we find $B \in X$.

If $(t, B)=(\operatorname{up}(A), A)$, then $B=A$, and $B \in X$ follows by Property 2 for $X$.

(b) $t=r \cdot C s$. Then $\{(s, C),(r, C \rightarrow B)\} \subseteq \mathcal{E}\left(\mathcal{B}^{A}\right)$ by Lemma 4.2. By IH we find $\{C, C \rightarrow B\} \subseteq X$. By Property 1 for $X$ we know that $C \in X$ and $C \rightarrow B \in X$ imply $B \in X$. Hence, we conclude that $B \in X$.

Remark 28. Gärdenfors [17] presented two more postulates that in our context read as

1. if $A \in \square_{\mathcal{M}}$, then $\square_{\mathcal{M}}=\square_{\mathcal{M}} \oplus A$

2. if $\square_{\mathcal{M}} \subseteq \square_{\mathcal{M}^{\prime}}$, then $\square_{\mathcal{M}} \oplus A \subseteq \square_{\mathcal{M}^{\prime}} \oplus A$.

It is standard [16] to show that these two additional postulates follow from the properties established in Lemma 27. 


\section{Ramsey Axiom}

The Ramsey axiom makes it possible to express the beliefs after an update in terms of the beliefs before the update. In dynamic doxastic logic, for example, Segerberg [24] formulates the Ramsey axiom as

$$
[A] \square B \leftrightarrow \square(A \rightarrow B) .
$$

Thus, it states that an agent believes $B$ after an update with $A$ if and only if the agent believes that $A$ implies $B$ before the update.

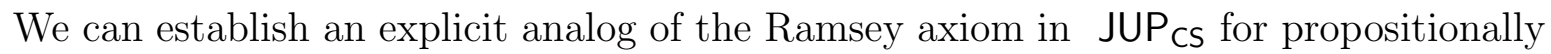
appropriate constant specifications.

We have to show the two implications of the Ramsey axiom separately. First, the direction from right to left.

Lemma 29 (Ramsey I). JUP ${ }_{\mathrm{CS}} \vdash t:(A \rightarrow B) \rightarrow[A] t \cdot{ }_{A}$ up $(A): B$.

Proof. Let $\mathcal{M}$ be an initial CS-model. Assume $\mathcal{M} \Vdash t:(A \rightarrow B)$ for some term $t$. By axiom (Pers) and soundness, $\mathcal{M} \Vdash[A] t:(A \rightarrow B)$. Moreover, using axiom (Up) we find $\mathcal{M} \Vdash[A] \operatorname{up}(A): A$ and by (MC.2) we obtain $\mathcal{M} \Vdash[A] t \cdot{ }_{A}$ up $(A): B$. The claim follows by completeness.

The direction from left to right of (3) need not hold in general. For a simple counterexample consider the following. By (Up) we have JUPCS $\vdash[A] \operatorname{up}(A): A$. However, if the constant specification CS is not propositionally appropriate, e.g., for CS $=\varnothing$, any model $\mathcal{M}=(\mathrm{v}, \varnothing)$ is an initial $\varnothing$-model. It is easy to see that $\mathcal{E}(\varnothing)=\varnothing$ and $\mathcal{M} \nVdash t: B$ for any term $t$ and any formula $B$. By completeness, JUP $\varnothing t:(A \rightarrow A)$.

For a propositionally appropriate constant specification, we do have an explicit version of the direction from left to right.

Lemma 30 (Ramsey II). Let CS be a propositionally appropriate constant specification. For each term there exists a term s such that

$$
\mathrm{JUP}_{\mathrm{CS}} \vdash[A] t: B \rightarrow s:(A \rightarrow B)
$$

Proof. Let $\mathcal{M}$ be an initial CS-model with $\mathcal{M} \Vdash[A] t: B$. By induction on the structure of $t$ we show $\mathcal{M} \Vdash s:(A \rightarrow B)$ for some $s$, then (4) follows by completeness. We distinguish the following cases for $t$ :

1. $t \in \mathrm{ATm}$. There are two possibilities:

- $t \neq u$ up $(B)$ or $A \neq B$. By the axiom of minimal change (MC.1) we find $\mathcal{M} \Vdash t: B$. Since CS is propositionally appropriate, there exists a constant $c$ such that $\mathcal{M} \Vdash$ $c:(B \rightarrow(A \rightarrow B))$. Hence, we conclude $\mathcal{M} \Vdash c \cdot{ }_{B} t:(A \rightarrow B)$.

- $t=\operatorname{up}(B)$ and $A=B$. Then there is a constant $c$ such that $\mathcal{M} \Vdash c:(A \rightarrow B)$ since CS is propositionally appropriate. 
2. $t=r \cdot{ }_{C} s$. It follows by (MC.2) that $\mathcal{M} \Vdash[A] r:(C \rightarrow B)$ and $\mathcal{M} \Vdash[A] s: C$. By IH we get $\mathcal{M} \Vdash r^{\prime}:(A \rightarrow(C \rightarrow B))$ and $\mathcal{M} \Vdash s^{\prime}:(A \rightarrow C)$ for some terms $r^{\prime}$ and $s^{\prime}$. Since CS is propositionally appropriate, there exists a constant $c$ such that

$$
\mathcal{M} \Vdash c:((A \rightarrow(C \rightarrow B)) \rightarrow((A \rightarrow C) \rightarrow(A \rightarrow B))) .
$$

Thus, we find $\mathcal{M} \Vdash\left(c \cdot A \rightarrow(C \rightarrow B) r^{\prime}\right) \cdot{ }_{A \rightarrow C} s^{\prime}:(A \rightarrow B)$.

\section{Conclusion}

We have introduced JUP $_{C S}$, a justification logic for belief expansion. The explicit evidence

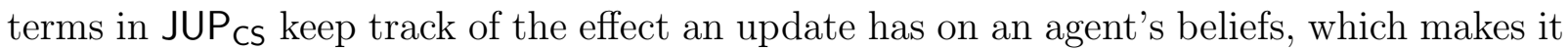
possible to axiomatize in the object language the principle of minimal change and establish soundness and completeness.

There are two directions of further research. One is to study belief contraction and revision in the context of justification logic. This is closely related to [22] where evidence elimination is studied.

A second line of research is to consider introspective agents. It is straightforward to add

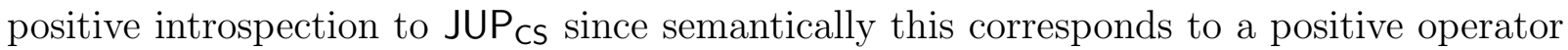
and, therefore, the least fixed point construction of the evidence relation still works. However, he properties with respect to belief sets and the Ramsey axiom will be different and, not surprisingly, the Moore's paradox will reappear. Adding negative introspection is also possible. The non-monotone inductive definitions used in the model constructions [25] for negative introspection provide a short preview of its belief dynamics.

\section{References}

1. C. E. Alchourrón, P. Gärdenfors, and D. Makinson. On the logic of theory change: Partial meet contraction and revision functions. Journal of Symbolic Logic, 50(2):510-530, 1985.

2. S. N. Artemov. Operational modal logic. Technical Report MSI 95-29, Cornell University, Dec. 1995.

3. S. N. Artemov. Explicit provability and constructive semantics. Bulletin of Symbolic Logic, 7(1):1-36, Mar. 2001.

4. S. N. Artemov. Kolmogorov and Gödel's approach to intuitionistic logic: current developments. Russian Mathematical Surveys, 59(2):203-229, 2004. Originally published in Russian.

5. S. N. Artemov. Justified common knowledge. Theoretical Computer Science, 357(1-3):4-22, July 2006.

6. S. N. Artemov. The logic of justification. The Review of Symbolic Logic, 1(4):477-513, Dec. 2008.

7. S. N. Artemov. Tracking evidence. In A. Blass, N. Dershowitz, and W. Reisig, editors, Fields of Logic and Computation, volume 6300 of LNCS, pages 61-74. Springer, 2010.

8. S. N. Artemov. The ontology of justifications in the logical setting. Studia Logica, 100(1-2):17-30, Apr. 2012. Published online February 2012.

9. S. N. Artemov and R. Kuznets. Logical omniscience as a computational complexity problem. In A. Heifetz, editor, TARK 2009, pages 14-23, Stanford University, California, July 6-8, 2009. ACM.

10. A. Baltag, B. Renne, and S. Smets. The logic of justified belief change, soft evidence and defeasible knowledge. In L. Ong and R. de Queiroz, editors, WoLLIC 2012, volume 7456 of LNCS, pages 168-190. Springer, 2012.

11. J. Barwise. Admissible Sets and Structures. Springer, 1975.

12. S. Bucheli, R. Kuznets, B. Renne, J. Sack, and T. Studer. Justified belief change. In X. Arrazola and M. Ponte, editors, LogKCA-10, Proceedings of the Second ILCLI International Workshop on Logic and Philosophy of Knowledge, Communication and Action, pages 135-155. University of the Basque Country Press, 2010. 
13. S. Bucheli, R. Kuznets, and T. Studer. Justifications for common knowledge. Journal of Applied Non-Classical Logics, 21(1):35-60, Jan.-Mar. 2011.

14. S. Bucheli, R. Kuznets, and T. Studer. Partial realization in dynamic justification logic. In L. D. Beklemishev and R. de Queiroz, editors, WoLLIC 2011, volume 6642 of LNAI, pages 35-51. Springer, 2011.

15. S. Bucheli, R. Kuznets, and T. Studer. Realizing public announcements by justifications. Journal of Computer and System Sciences, To appear.

16. H. van Ditmarsch, W. van der Hoek, and B. Kooi. Dynamic Epistemic Logic. Springer, 2007.

17. P. Gärdenfors. Knowledge in Flux. The MIT Press, 1988.

18. J. Gerbrandy and W. Groeneveld. Reasoning about information change. Journal of Logic, Language and Information, 6(2):147-169, Apr. 1997.

19. R. Kuznets and T. Studer. Justifications, ontology, and conservativity. In T. Bolander, T. Braüner, S. Ghilardi, and L. Moss, editors, Advances in Modal Logic, volume 9, pages 437-458. College Publications, 2012.

20. A. Mkrtychev. Models for the logic of proofs. In S. Adian and A. Nerode, editors, LFCS'97, volume 1234 of LNCS, pages 266-275. Springer, 1997.

21. B. Renne. Dynamic Epistemic Logic with Justification. PhD thesis, CUNY Graduate Center, May 2008.

22. B. Renne. Evidence elimination in multi-agent justification logic. In A. Heifetz, editor, TARK 2009, pages 227-236, Stanford University, California, July 6-8, 2009. ACM.

23. B. Renne. Public communication in justification logic. Journal of Logic and Computation, 21(6):1005-1034, Dec. 2011. Published online July 2010.

24. K. Segerberg. Belief revision from the point of view of doxastic logic. Logic Journal of the IGPL, 3(4):535-553, 1995.

25. T. Studer. Decidability for some justification logics with negative introspection. Journal of Symbolic Logic, To appear. 